\title{
Gestión de capital de trabajo y la toma de decisiones en la empresa alba mayo S.R.L, Moyobamba - 2021
}

\author{
Br. Yacseni Guerrero Altamirano \\ gguerrero@ucvvirtual.edu.pe \\ https://orcid.org/0000-0002-4371-5132
}

Br. Kevin Huamán Chuquipa

khuaman@ucvvirtual.edu.pe https://orcid.org/0000-0002-7755-5405

Dr. Juan Britman Vallejos Tafur https://orcid.org/0000-0002-6328-806X jvallejost@ucv.edu.pe

Universidad César Vallejo Moyobamba - Perú

\section{RESUMEN}

El objetivo general de la investigación fue determinar la relación que existe entre la gestión de capital de trabajo y la toma de decisiones en la empresa Alba Mayo S.R.L ubicado en la ciudad de Moyobamba, año 2021. El tipo de investigación es básica, su nivel es correlacional, diseño no experimental, con enfoque cuantitativo, de tipo aplicada, la población estuvo compuesta por 15 trabajadores de la empresa. Los resultados mostraron que el valor del coeficiente de Pearson obtenido es de 0.353 una correlación positiva baja y de sig., es igual a 0,000, también según el análisis financiero se obtuvo que el capital de trabajo para el 2019 la empresa dispuso de un capital de trabajo de s/.1,787,765.00 y para 2020 fue de 1,822,845, llegando a concluir que si existe relación entre la gestión de capital y toma de decisiones, pues mediante los análisis financieros y la utilización correcta de los ratios, permitirá a la empresa tomar decisiones.

Palabras claves: gestión de capital de trabajo; toma de decisiones; estados financieros. 


\title{
Working capital management and decision making in the company alba mayo S.R.L, Moyobamba - 2021
}

\begin{abstract}
The general objective of the research was to determine the relationship that exists between the management of working capital and decision-making in the company Alba Mayo SRL located in the city of Moyobamba, year 2021. The type of research is basic, its level is correlational, non-experimental design, with a quantitative approach, applied type, the population consisted of 15 company workers. The results showed that the value of the Pearson coefficient obtained is 0.353 , a low positive correlation and sig., Is equal to 0.000 , also according to the financial analysis it was obtained that the working capital for 2019 the company had a capital of work of s /. 1,787,765.00 and for 2020 it was 1,822,845, reaching the conclusion that if there is a relationship between capital management and decision-making, because through financial analysis and the correct use of ratios, it will allow the company to make decisions.
\end{abstract}

Keywords: working capital management; decision making; financial statements.

Artículo recibido: 15 noviembre. 2021 Aceptado para publicación: 10 diciembre 2021 Correspondencia: gguerrero@ucvvirtual.edu.pe Conflictos de Interés: Ninguna que declarar 


\section{INTRODUCCIÓN}

La gestión de capital de trabajo es necesario en una empresa por el número considerable de actividades que se realizan es también un fondo de la cual utilizan la empresa para poder garantizar la rentabilidad esperada, pues la valoración de la administración del capital de trabajo, consentirá tramitar eficientemente cada uno de los recursos económicos que tiene, mediante este procedimiento mejorar la fluctuación de los recursos y por ende mejorar las condiciones y ganancias de las empresas.

La problemática de esta investigación es la ineficiente gestión de capital de trabajo en la toma de decisiones en la empresa Alba Mayo SRL, Moyobamba 2021, dado que debe afrontar el problema de la gestión de los recursos, carece de estrategias para gestionar adecuadamente el capital de trabajo, por lo que no está preparada para afrontar nuevos retos ni tomar decisiones trascendentes y de gran magnitud, del mismo modo hay diferentes empresas que enfrentan problemas financieros, como el insuficiente liquidez, baja rentabilidad, problemas de financiamiento, retrasos en los pagos a los proveedores. En el ámbito internacional, Jaramillo 2018, en su investigación sobre la relación entre la gestión de capital de trabajo y la rentabilidad en las empresas de la industria de distribución de químicos en Colombia el cual agrupó a 48 empresas del sector, remarcó que la rentabilidad de las empresas puede mejorar de acuerdo con el manejo eficiente del capital de trabajo, así mismo puede mejorar la liquidez para invertir o apalancarse. Y esto a largo plazo se ve reflejado en la mejora de la situación económica y financiera de las empresas. En el ámbito nacional, Pusma, et al. En 2020, en su investigación sobre la gestión de capital de trabajo como táctica económica para la mejora institucional, mencionan que el capital de trabajo ayuda a estabilizar la liquidez, la economía y la viabilidad en la mayoría de las empresas. Para ello, deben evaluar lo siguiente, minimizar el tiempo de cobro de las cuentas por cobrar. Por siguiente, aumentar el proceso productivo de materia prima en productos terminados y ventas posteriores, por tercero pagar las facturas que no han sido canceladas lo más pronto posible, por lo que cada empresa debe generar ingresos mayores a sus gastos, esto requiere de una gestión eficaz del pasivo corriente, activo, mediante de una buena planificación, ejecución, reducción y control para ampliar la rentabilidad de la empresa teniendo en cuenta los riesgos. 


\section{A. Trabajos previos}

Para el desarrollo del estudio se analizó en el contexto internacional y nacional diversas investigaciones relacionadas con las variables a estudiar, siendo de gran utilidad para discutir los resultados futuros de la investigación tales como las realizadas por Silvestre (2021). En su investigación determino la relación que existe entre capital de trabajo y ventaja competitiva de la empresa, tuvo como resultado que en la empresa el $62.5 \%$ de los colaboradores perciben al capital de trabajo como bueno por ende la empresa es eficiente, con respeto a la ventaja competitiva el $62.5 \%$ encuestados refieren que la empresa si tiene una ventaja competitiva. Aguilar (2020). Determino el efecto de reflexionar sobre los diferentes estilos de toma de decisiones en las pequeñas y medianas empresas de la sección de elaboración de fabricado en la ciudad de Quito. El estilo dialéctico, el estilo adicto e intuitivo comparecen a ser secundarios entretanto que el estilo automático no influye de representación demostrativa en la forma de toma de decisiones, el cual mostro que $43 \%$ de las empresas pequeñas de Quito Ecuador se considera que los mandos gerenciales toman buenas decisiones mientras que sólo un $15 \%$ de las medianas empresas del mismo sector tienen una deficiencia en la toma de decisiones repercutiendo en la rentabilidad de las mismas. Malquin et al. (2019). El objetivo de la investigación fue estudiar y evaluar el estado del capital de trabajo para la empresa la empresa Transcomerinter de la ciudad de Tulcán. Después de analizar los resultados se determinó que la empresa tiene la necesidad de establecer un presupuesto de capital de trabajo que contribuya al desarrollo y mejoramiento de la empresa, ya que se encuentra en una importante crisis con respecto al capital de trabajo debido a un mal manejo de los directivos. Peña et al. (2019). El análisis financiero permite conocer las principales deficiencias de una empresa y que a su vez ayuda a tomar decisiones útiles y oportunas para el mejoramiento de la misma. Rosell. (2019). Los resultados evidenciaron que el análisis de los estados financieros tiene mucha relación con la toma de decisiones ya que ambos permiten a la empresa crecer económicamente y financieramente. Sanabria et al. (2017). La toma de decisiones en una empresa es un factor importante para mejorar la productividad y las ganancias, esto se puede llevar mediante estrategias que implementen los directivos de la empresa. Rivillas et al. (2017). La mayoría de las empresas no realizan diagnósticos financieros o se limitan a aspectos básicos, además, se puede apreciar que la principal dificultad está relacionada con la rentabilidad, lo que afecta la sostenibilidad a 
medio y largo plazo. Así mismo el análisis efectuado con los indicadores financieros muestran que las empresas no cuentan con los recursos necesarios para cubrir obligaciones corrientes, ya que el $70 \%$ es en deuda con proveedores a corto plazo, y esta situación requiere de atención dado que afecta la liquidez para atender las obligaciones a corto plazo.

De forma similar se hizo uso de investigaciones nacionales para profundizar el estudio de la investigación. Siancas (2020). estudio de los estados financieros y su incidencia en la toma de resoluciones corporation intherpharma S.A.C, revelan que los directivos toman decisiones sin hacer su respectivo análisis de ningún método a los estados financieros, esto muestra que para el año 2019 los gastos administrativos aumentaron a $52.75 \%$ y las ventas disminuyeron un $3.65 \%$. Paola. (2019). La influencia que tiene el capital de trabajo en la ventaja competitiva muestra que el capital de trabajo posee un elevado nivel de importancia en el beneficio de las empresas de ferreterías y que en muchas desconocen el buen manejo de un capital de trabajo. Chaves et al. (2017). En su investigación, gestión de la información financiera y su relación con la toma de decisiones gerenciales en las organizaciones de la Unión Peruana del Norte, se muestra la relación entre servicio al cliente y la ventaja de toma de decisiones y que la gestión de la información financiera es muy importante para la toma de decisiones.

\section{B. Teorías relacionadas al tema}

Hemos definido variables y dimensiones. Para Ortiz, H. (2006), el conocimiento diferente es muy importante para la gestión de capital de trabajo, y es la base para que los activos líquidos reflejen el saldo global de sus activos; difundir activamente el repertorio, cuentas por cobrar, el seguimiento y la capacitación de los activos corrientes, como las cuentas, fluctúa de manera eficiente; esto es muy importante en las organizaciones de rápido crecimiento porque no existe una capacitación de transformación bien documentada en estos activos. Para las empresas, su financiamiento externo es el pasivo corriente, por lo que la puerta al mercado en periodos de altos costos se limita a ellas.

Según Van Horne y Wachowicz (2016), el capital de trabajo se puede dividir en dos tipos: a) Según el efectivo de su equipo, naturaleza transferible, saldo por cobrar e inventario. b) Desde el nombramiento hasta el plazo. Capital de comisión local, es la simetría del activo circulante para hacer frente a la pequeña disposición de la espaciosa división. Es decir, es el pago de la liquidación que mantiene un año de hospitalidad, 
recogido por clichés, repertorios y cuentas. Capital de deuda territorial, es el cambio de activo circulante requerido en los tiempos.

Las Dimensiones: Según Lira (2016) planea las siguientes con relación de la gestión del Capital de trabajo Activo corriente: El activo corriente, igualmente designado como activo líquido o circulante, es el activo de la organización que consigue concebir líquido, es decir, que puede cristianizar en capital, en un término de tiempo menor a un año. El pasivo corriente, también propuesto como pasivo circulante, es el que está desarrollado por los deberes a corto plazo.

En relación a la versátil toma de decisiones Mallo, C. y Rocafort, A. (2016), "la toma de decisiones es un asunto usual en el que los representantes de una empresa se afrontan cada vez, teniendo asimismo una de los compromisos más dificultosos. Presume la deliberación de la principal disyuntiva entre las efectivas para brindar el mejor procedimiento a un problema de gestión. Al mismo tiempo, Vega, Vivanco, y Herrera (2016) lo definen como "el proceso para la identificación y solución de problemas específicos" (p. 13). Es decir, es el proceso a través del cual se considera una opción, clara, informada y motivada. Permitiendo elegir entre varias opciones la mejor forma posible de actuar con el propósito de alcanzar un objetivo, en determinadas condiciones y circunstancias.

Koontz, et al. 2017 sugirieron que la médula de la planeación es la toma de decisiones, y obliga como la elección en un ámbito de atribución de diversas disyuntivas. La cual no expresa que preexiste un proyecto, dado que se haya tomado una decisión arriesgada poniendo en peligro los capitales, solo en ese momento se puede hacer una planeación y observación del problema.

Respecto a la teoría de Guzmán (2018) la toma de decisiones representa un proceso estratégico, que busca analizar, seleccionar y optar por más alternativas de solución a los problemas. En muchas oportunidades, los gerentes creen que es su modelo de servicio tener poder de decisión, porque generalmente ellos deciden a qué acudir, quién lo hará, cuándo e incluso cómo se debe resolver, sin embargo, la toma de decisiones es solo planificación y razonamiento. Así, de la misma forma cuando una persona actúa rápidamente sin detalles extremos, o cuando una acción tiene solo unos minutos de privilegio, el plan sigue siendo el mismo. Es una referencia a la vida diaria de todos. Los 
periodos raros pueden definir una sola capa de movimiento, porque implícitamente cada decisión debe adaptarse al nuevo procedimiento.

Este proceso involucra una serie de actos parciales y secuenciales que permitirán a lo que les impide tomar decisiones correctas, sumado a ello la persona desde su conciencia hasta la necesidad elegir una solución entre las más correctas, considerando la situación y pasando por la recogida y tratamiento de la información importante. Respecto a la teoría de Guzmán (2018) la toma de decisiones representa un proceso estratégico, que busca analizar, seleccionar y optar por más alternativas de solución a los problemas. En definitiva, se determina que la ventaja de disposiciones es un factor crítico dentro de los negocios, pues sugieren la necesidad de optar entre varias alternativas como una técnica para afrontar los problemas (Yeong, Choi, \& Watabe, 2020). Por lo tanto, Guzmán (2018) determina que la toma de decisiones debe evaluarse a través de dos componentes, como el proceso de toma de decisiones y los tipos de decisiones tomadas en la empresa. Dado ello, el proceso de toma de decisiones involucra el diagnóstico del problema, la identificación de criterios para ponderar las decisiones, priorizar la atención del problema identificado, además, es necesario formular, valorar y elegir la mejor alternativa de solución, la misma que debe ser implementada y evaluada posteriormente.

Asimismo, Guzmán (2018) considera que los tipos de decisión están centrados en las inversiones que requiera la empresa o sean necesarias para la misma, así como las providencias de financiamiento que busquen a un desarrollo de la compañía.

\section{ESTRATEGIAS METODOLÓGICAS O MATERIALES Y MÉTODOS}

En esta investigación se consideró como objetivo general determinar la relación entre gestión de capital de trabajo y la toma de decisiones en la empresa Alba Mayo SRL, Moyobamba, 2021. Este trabajo es de tipo aplicada ya que se refleja en la aplicación de conocimientos y resultados por autores anteriores en diagnosticar, evaluar y presentar posibles soluciones a los problemas para el mejor desarrollo de nuestro proyecto, así mismo aplicar nuestros conocimientos basados en la realidad de la empresa y nuestra experiencia desarrollando esto de manera detallada y ordenada. Pues de acuerdo a Sánchez (2018), se le denomina aplicada puesto que, se tomarán bases teóricas que serán aplicadas para la evaluación de cada una de las variables. Así mismo el nivel de la investigación fue correlacional, pues se mide la relación entre dos variables sin que el investigador controle ninguna de ellas, es así que a través del estudio se pretendió 
determinar la relación entre el capital de trabajo y la toma de decisiones en la empresa Alba Mayo S.R.L, en el año 2021, procedimiento que fue contrastada de forma estadística por (Hernández et al. 2018). También el diseño de investigación fue no experimental, pues se realizó sin manipular de forma deliberada las variables y se observó los fenómenos en su ambiente natural para después ser analizados (Hernández, 2018).

La muestra estuvo conformada por 15 trabajadores de la empresa de distintas áreas de la empresa, así mismo se desarrollo en base a los estados financieros de los años 2019 y 2020. Las técnicas utilizadas fueron la encuesta y el análisis de documental. El método empleado para el análisis de datos fue el estadístico pues se realizó mediante los programas Microsoft Excel e IBM SPSS.

\section{RESULTADOS Y DISCUSIÓN}

\section{A. Resultado inferencial}

\section{Tabla 1}

Relación de la gestión de capital de trabajo en la toma de decisiones

\begin{tabular}{llcc}
\hline & & Gestión de capital & $\begin{array}{c}\text { Toma de } \\
\text { decisiones }\end{array}$ \\
\hline \multirow{3}{*}{ Gestión capital } & Correlación de Pearson & 1 &, 353 \\
& Sig. (bilateral) & &, 001 \\
& $\mathrm{~N}$ & 15 & 15 \\
Toma de & Correlación de Pearson &, 353 & 1 \\
decisiones & Sig. (bilateral) &, 001 & 15 \\
\hline
\end{tabular}

En la tabla 1 se contempla la relación entre la gestión de capital de trabajo y la toma de decisiones en la empresa Alba Mayo SRL, Moyobamba, 2021. Mediante el análisis estadístico de coeficiente de Pearson se alcanzó un coeficiente de 0.353 (correlación positiva baja) y un $\mathrm{p}$ valor igual a $0,000(\mathrm{p}$-valor $\leq 0.01)$ en todas las correlaciones, por lo que, se acepta la hipótesis alterna, es decir, existe relación entre la gestión de capital de trabajo y la toma de decisiones en la empresa Alba Mayo SRL, Moyobamba, 2021.

\section{Tabla 2}

Influye favorablemente el Nivel del capital de trabajo en la toma de decisiones

\begin{tabular}{ccc}
\hline Escala & Frecuencia & Porcentaje \\
\hline $\mathrm{Si}$ & 8 & $53 \%$ \\
$\mathrm{No}$ & 7 & $47 \%$ \\
Total & 15 & $100 \%$ \\
\hline
\end{tabular}

Fuente: Cuestionario aplicado a los trabajadores en la empresa Alba Mayo SRL, Moyobamba, 2021. 
En la tabla 2 respecto al nivel de capital de trabajo, el $53 \%$ manifestó que si existe un buen capital de trabajo y el $47 \%$ indicaron que no existe un buen capital de trabajo.

\section{Tabla 3}

Influye favorablemente el nivel de activos corrientes y pasivos corrientes en la toma de decisiones

\begin{tabular}{lcc}
\hline \multicolumn{1}{c}{ Capital de trabajo } & \multicolumn{2}{c}{ Toma de decisiones } \\
& correlación & Sig. \\
\hline Activos corrientes & $0.379^{* *}$ & 0.000 \\
Pasivo corriente & $0.328^{* *}$ & 0.000 \\
\hline
\end{tabular}

La correlación es significativa en el nivel 0,01 (bilateral).

En la tabla 3 se contempla la influencia de los activos y pasivos corrientes en la toma de decisiones de la empresa Alba Mayo SRL, Moyobamba, 2021. Mediante el análisis estadístico de Pearson se alcanzó un coeficiente de 0.379 y 0.328 (correlación positiva baja) y un p valor igual a 0,000 (p-valor $\leq 0.01$ ) en todas las correlaciones, por lo que, se acepta la hipótesis alterna, es decir, el nivel de los activos y pasivos corrientes influye favorablemente en la toma de decisiones en la empresa Alba Mayo SRL, Moyobamba, 2021.

\section{Tabla 4}

Influencia de los estados financieros en la toma de decisiones

\begin{tabular}{|c|c|c|c|}
\hline & & Estados financieros & Toma de decisiones \\
\hline \multirow{3}{*}{$\begin{array}{l}\text { Estados } \\
\text { financieros }\end{array}$} & $\begin{array}{l}\text { Correlación de } \\
\text { Pearson }\end{array}$ & 1 & ,323 \\
\hline & Sig. (bilateral) & &, 001 \\
\hline & $\mathrm{N}$ & 15 & 15 \\
\hline \multirow{3}{*}{$\begin{array}{l}\text { Toma } \\
\text { decisiones }\end{array}$} & $\begin{array}{l}\text { Correlación de } \\
\text { de Pearson }\end{array}$ & ,323 & 1 \\
\hline & Sig. (bilateral) & ,001 & \\
\hline & $\mathrm{N}$ & 15 & 15 \\
\hline
\end{tabular}

En la tabla 4 se contempla la influencia de los estados financieros en la toma de decisiones de la empresa Alba Mayo SRL, Moyobamba, 2021. Mediante el análisis estadístico de Pearson se alcanzó un coeficiente de 0.323 (correlación positiva baja) y un p valor igual a 0,000 (p-valor $\leq 0.01$ ) en todas las correlaciones, por lo que, se acepta la hipótesis alterna, es decir, el análisis de los estados financieros influye favorablemente en la toma de decisiones en la empresa Alba Mayo SRL, Moyobamba, 2021. 


\section{B. Resultado descriptivo}

\section{Análisis de ratios}

Para analizar la situación económica de la empresa, se realizó un análisis de ratios de los estados financieros del periodo 2019 y 2020.

\section{Tabla 5}

Ratio de capital de trabajo

\begin{tabular}{l} 
Capital de Trabajo \\
\hline$\underline{\text { Periodo } 2019}$ \\
Activo corriente - Pasivo corriente \\
$2,639,549-851,784$ \\
\hline Resultado $=\quad \mathbf{1 , 7 8 7 , 7 6 5 . 0 0}$ \\
\hline
\end{tabular}

\begin{tabular}{l}
\multicolumn{1}{c}{ Capital de Trabajo } \\
\hline$\underline{\text { Periodo 2020 }}$ \\
Activo corriente - Pasivo corriente \\
$6,725,997-4,903,152$ \\
Resultado $=\mathbf{1 , 8 2 2 , 8 4 5}$ \\
\hline
\end{tabular}

En la tabla 5 se muestra que en el periodo 2019 la empresa dispuso de un capital de trabajo de s/.1,787,765.00 y para el año 2020 obtuvo de s/. 1,822,845 produciéndose una mejora significativa a fin de cumplir con los compromisos que origina la empresa.

\section{Tabla 6}

Ratio de liquidez corriente

\begin{tabular}{c} 
Liquidez Corriente \\
\hline$\frac{\text { Periodo 2019 }}{\text { activo corriente }}$ \\
\hline pasivo corriente \\
$2,639,549$ \\
851,784 \\
Resultado $=$ \\
\hline
\end{tabular}

\begin{tabular}{c} 
Liquidez Corriente \\
\hline$\underline{\text { Periodo 2020 }}$ \\
activo corriente \\
\hline Pasivo corriente \\
$6,725,997$ \\
$4,903,152$ \\
Resultado $=\quad 1.37$ \\
\hline
\end{tabular}

En la tabla 6 se muestra el indicador de liquidez para el año 2019 tiene un buen indicador es decir que por cada S/. 1.00 de deuda a corto plazo, la empresa tiene S/. 3.1 soles para cubrirlos con sus activos de corto plazo y para el año 2020 la empresa por cada S/.1.00, la empresa tiene S/.1.37 soles para cubrir con sus activos de corto plazo. 


\section{Tabla 7}

Ratio de margen comercial

\begin{tabular}{c} 
Margen Comercial \\
\hline $\begin{array}{c}\text { Periodo } 2019 \\
\text { ventas netas - costo de ventas }\end{array}$ \\
ventas netas \\
$21,495,589-20,139,248$ \\
$21,495,589$ \\
Resultado $=0.06 \quad 6 \%$ \\
\hline
\end{tabular}

\begin{tabular}{c} 
Margen Comercial \\
\hline Periodo 2020 \\
ventas netas - costo de ventas \\
ventas netas \\
$25,862,513-24,296,524$ \\
\hline $25,862,513$ \\
Resultado= $0.06 \quad 6 \%$ \\
\hline
\end{tabular}

En la tabla 7 se puede observar que del periodo 2019 al periodo 2020, podemos interpretar que por cada s/.1.00 invertido la empresa genera una ganancia del $6 \%$ para el periodo 2019 y $6 \%$ para el 2020 por lo que se analiza que de un periodo a otro ha habido un incremento de gastos en la producción de venta.

\section{Tabla 8}

Ratio de Endeudamiento

\section{Endeudamiento a corto plazo}

Periodo 2019

$\frac{\text { Pasivo corriente }}{\begin{array}{c}\text { Patrimonio } \\ \text { neto }\end{array}}$

851,784

$2,635,248$

Resultado $=0.32$
Endeudamiento a corto plazo

Periodo 2020

\begin{tabular}{cc}
\hline $\begin{array}{c}\text { Pasivo corriente } \\
\text { Patrimonio } \\
\text { neto } \\
4,903,152 \\
\end{array}$ \\
Resultado $=$ & 1.53 \\
\hline
\end{tabular}

En la tabla 8 se refleja el rendimiento del dinero invertido por el dueño de la empresa, en esta ratio podemos analizar la dependencia de la empresa sobre capitales propios o el uso de capitales de terceros para el cumplimiento de sus obligaciones y sus planes de inversión. Los resultados indican que en el periodo 2019 el resultado fue de 0.32 y para el periodo 20201.53 teniendo un incremento de 1.21 esto quiere decir que con el financiamiento propio y las obligaciones que aportan, se ha conseguido el 1.21 de esta inversión de un periodo al otro. 


\section{Tabla 9}

Ratio de rentabilidad sobre el patrimonio.

\begin{tabular}{c} 
Rentabilidad sobre el patrimonio \\
\hline \hline \begin{tabular}{c} 
Periodo 2019 \\
Utilidad neta \\
\hline Patrimonio \\
968,158 \\
\hline $3,487,248$
\end{tabular}
\end{tabular}

Resultado $=0.28 \quad 28 \%$

\section{Rentabilidad sobre el patrimonio}

Periodo 2020

Utilidad neta

Patrimonio

879,744

$3,211,654$

Resultado $=0.27$

$27 \%$

En la tabla 9 se contempla con los valores mínimos y máximo observado en cada ratio de rentabilidad dentro del periodo analizado, se tiene que el promedio del ratio de rentabilidad sobre patrimonio para el periodo 2019 fue de 0.28 y 0.27 para el 2020, nos indica que para el periodo 2019 se obtuvo una ganancia de $28 \%$ sobre la inversión realizada y para el 2020 un $27 \%$, lo que demuestra una decreciente rentabilidad sobre el patrimonio.

\section{Análisis horizontal y vertical de los estados financieros}

Para mayor análisis de la situación de la empresa para la toma de decisiones se presentan el análisis horizontal y vertical de los estados financieros tales como: Estado de situación financiera y estado de resultado integral de la empresa Alba Mayo SRL del periodo 2019 y 2020.

\section{Tabla 10}

Análisis vertical del activo

\begin{tabular}{lcccc}
\hline ACTIVO CORRIENTE & $\mathbf{2 0 1 9}$ & $\mathbf{\%}$ & $\mathbf{2 0 2 0}$ & $\boldsymbol{\%}$ \\
\hline Caja y bancos & 702,329 & $20 \%$ & $4,668,625$ & $58 \%$ \\
Cuentas por cobrar & 618,685 & $18 \%$ & 305,448 & $4 \%$ \\
Mercadería & 683,204 & $20 \%$ & $1,422,777$ & $18 \%$ \\
Subproductos y desechos & 397,890 & $11 \%$ & 86,380 & $1 \%$ \\
Mercadería extraída & 237,441 & $7 \%$ & 242,767 & $3 \%$ \\
\hline Total activo corriente & $\mathbf{2 , 6 3 9 , 5 4 9 . 0 0}$ & $\mathbf{7 6 \%}$ & $\mathbf{6 , 7 2 5 , 9 9 7 . 0 0}$ & $\mathbf{8 3 \%}$ \\
\hline
\end{tabular}




\begin{tabular}{lcccc}
\hline ACTIVO NO CORRIENTE & $\mathbf{2 0 1 9}$ & $\mathbf{\%}$ & $\mathbf{2 0 2 0}$ & $\mathbf{\%}$ \\
\hline Inmuebles, maquinaria y equipo & 578,709 & $17 \%$ & $1,118,574$ & $14 \%$ \\
Depreciación acumulada & $-51,023$ & $1 \%$ & $-105,104$ & $1 \%$ \\
Intangibles & 2,000 & $0 \%$ & 2,000 & $0 \%$ \\
Activo diferido & & $0 \%$ & & $0 \%$ \\
Activos no corrientes & 317,797 & $9 \%$ & 373,339 & $5 \%$ \\
\hline Total activos no corrientes & $\mathbf{8 4 7 , 4 8 3}$ & $\mathbf{2 4 \%}$ & $\mathbf{1 , 3 8 8 , 8 0 9}$ & $\mathbf{1 7 \%}$ \\
\hline Total activo neto & $\mathbf{3 , 4 8 7 , 0 3 2}$ & $\mathbf{1 0 0 \%}$ & $\mathbf{8 , 1 1 4 , 8 0 6}$ & $\mathbf{1 0 0 \%}$ \\
\hline
\end{tabular}

Se puede evidenciar en el estado de situación financiera que el año 2019 la empresa se financió a corto plazo siendo así el activo corriente el 76\% del total de los activos y en el año 2020 también fue a corto plazo con el 83\% del total de los activos, la cuenta con mayor porcentaje dentro del activo corriente es caja y bancos para el año 2019 con el $20 \%$ y en el 2020 de $58 \%$, lo que significa que la empresa no hace uso de su dinero para inversiones. En lo referente al activo no corriente inversiones a largo plazo se puede observar que el mayor porcentaje se muestra en la cuenta de inmuebles, maquinaria y equipos para el año 2019 con el $17 \%$ del activo total y para el 2020 con el $14 \%$ del activo total.

\section{Tabla 11}

Análisis vertical del pasivo mas patrimonio

\begin{tabular}{lcccc}
\hline PASIVO & $\mathbf{2 0 1 9}$ & $\mathbf{\%}$ & $\mathbf{2 0 2 0}$ & $\mathbf{\%}$ \\
\hline Tributos y aportaciones por pagar & 36,812 & $1 \%$ & 2,440 & $0 \%$ \\
Remuneraciones por pagar & 5,600 & $0 \%$ & 1,023 & $0 \%$ \\
Anticipos a clientes & 298,428 & $9 \%$ & $-1,527$ & $0 \%$ \\
Cuentas por pagar comercial & 306,809 & $9 \%$ & 27,753 & $0 \%$ \\
Obligaciones financieras & 204,135 & $6 \%$ & $4,873,463$ & $60 \%$ \\
\hline Total pasivo & $\mathbf{8 5 1 , 7 8 4}$ & $\mathbf{2 4 \%}$ & $\mathbf{4 , 9 0 3 , 1 5 2}$ & $\mathbf{6 0 \%}$ \\
\hline PATRIMONIO & & & & \\
\hline Capital & 500,000 & $14 \%$ & 500,000 & $6 \%$ \\
Resultados acumulados & $1,167,090$ & $33 \%$ & $1,866,706$ & $23 \%$ \\
Utilidad del ejercicio & 968,158 & $28 \%$ & 844,948 & $10 \%$ \\
\hline Total patrimonio & $\mathbf{2 , 6 3 5 , 2 4 8}$ & $\mathbf{7 6 \%}$ & $\mathbf{3 , 2 1 1 , 6 5 4}$ & $\mathbf{4 0 \%}$ \\
\hline Total pasivo y patrimonio & $\mathbf{3 , 4 8 7 , 0 3 2}$ & $\mathbf{1 0 0 \%}$ & $\mathbf{8 , 1 1 4 , 8 0 6}$ & $\mathbf{1 0 0 \%}$ \\
\hline
\end{tabular}


Con respecto al financiamiento de la empresa por parte de terceros sus cuentas con mayor porcentaje para el año 2019 fueron las cuentas por cobrar con el 9\%, anticipos a clientes el 9\% y obligaciones con el 6\%. Por el contrario, en el año 2020 su partida más importante fue la cuenta obligaciones financieras con el $60 \%$ del total pasivo mas patrimonio. Por último, el patrimonio en el año 2019 fue del 76\% del total pasivo más patrimonio y en el 2020 de $40 \%$ del total pasivo mas patrimonio, sus partidas más relevantes fueron para el 2019, resultados acumulados del 33\% y utilidad del ejercicio con $28 \%$, para el año 2020 fueron resultados acumulados del $23 \%$ y utilidad del ejercicio con el $10 \%$.

\section{Tabla 12}

Análisis vertical del estado de resultado

\begin{tabular}{|c|c|c|c|c|}
\hline & 2019 & $\%$ & 2020 & $\%$ \\
\hline Ingresos de operación & $21,495,589$ & $100 \%$ & $25,862,513$ & $100 \%$ \\
\hline (-) Costo de ventas & $20,139,248$ & $94 \%$ & $24,296,524$ & $94 \%$ \\
\hline Resultado bruto (utilidad) & $1,356,341$ & $6 \%$ & $1,565,989$ & $6 \%$ \\
\hline \multicolumn{5}{|l|}{ Gastos de operación } \\
\hline Gastos de venta & 201,563 & $1 \%$ & 274,854 & $1 \%$ \\
\hline Gastos de administración & 181,233 & $1 \%$ & 410,777 & $2 \%$ \\
\hline Resultado de operación & $\mathbf{9 7 3 , 5 4 5}$ & $5 \%$ & 880,358 & $3 \%$ \\
\hline Gastos financieros & 37,621 & $0 \%$ & 119,476 & $0 \%$ \\
\hline Ingresos financieros & 12,033 & $0 \%$ & 48,447 & $0 \%$ \\
\hline Otros ingresos gravados & 20,200 & $0 \%$ & 35,620 & $0 \%$ \\
\hline Resultados antes de impuestos & 968,158 & $5 \%$ & 844,948 & $3 \%$ \\
\hline Distribución legal de la renta & & & 0 & \\
\hline Resultados antes de impuestos & 968,158 & & 844,948 & \\
\hline Adiciones para determinar la renta & & & 34,796 & $0 \%$ \\
\hline Adiciones para determinar la renta & & & 0 & \\
\hline
\end{tabular}

\begin{tabular}{lllll}
\hline Renta neta imponible & $\mathbf{9 6 8 , 1 5 8}$ & $\mathbf{0}$ & $\mathbf{8 7 9 , 7 4 4}$ & \\
\hline \hline & & & & \\
\hline Impuesto a la renta & $\mathbf{2 8 5 , 6 0 7}$ & $1 \%$ & $\mathbf{2 5 9 , 5 2 5}$ & $1 \%$ \\
\hline
\end{tabular}

En el año 2019 se observa que los costos de ventas ascienden monetariamente a S/ 20,139,248, que representa el $94 \%$ de las ventas, esto significa que la empresa tiene elevado los costos de ventas, observando el año 2020 se puede evidenciar que los costos 
de ventas fueron mayor a comparación con el año 2019, lo que significa que en el año 2020 la empresa no obtuvo un adecuado control de manejo de costos. Con relación al año 2019 los gastos de operación con mayor ascendente monetario son los gastos de venta de S/201,563 porcentualmente del 1\% y los gastos de administración de S/ 181,233 con un $1 \%$ porcentualmente. Con respecto al año 2020 los gastos de operación con mayor porcentaje se ven reflejado en los gastos administrativos con un $2 \%$ y los gastos de ventas con el 1\%. Por último, la renta neta imponible se muestra que en el año 2019 es del $1 \%$ del total de las ventas y en el año 2020 el $1 \%$ los cuales son porcentajes muy bajos para el nivel de ventas que tiene la empresa

\section{Tabla 13}

Análisis horizontal del estado de situación financiera

\begin{tabular}{|c|c|c|c|c|}
\hline & & & & 20 \\
\hline ACTIVO CORRIENTE & 2020 & 2019 & variación & Porcentaje \\
\hline Caja y bancos & $4,668,625$ & 702,329 & $3,966,296$ & $565 \%$ \\
\hline Cuentas por cobrar & 305,448 & 618,685 & $-313,237$ & $-51 \%$ \\
\hline Mercadería & $1,422,777$ & 683,204 & 739,573 & $108 \%$ \\
\hline Subproductos y desechos & 86,380 & 397,890 & $-311,510$ & $-78 \%$ \\
\hline Mercadería extraída & 242,767 & 237,441 & 5,326 & $2 \%$ \\
\hline Total activo corriente & 6725997 & 2639549 & $4,086,448$ & $155 \%$ \\
\hline ACTIVO NO CORRIENTE & 3487032 & 8114806 & -4627774 & $-57 \%$ \\
\hline Inmuebles, maquinaria y equipo & $1,118,574$ & 578,709 & 539,865 & $93 \%$ \\
\hline Depreciación acumulada & $-105,104$ & $-51,023$ & $-54,081$ & $106 \%$ \\
\hline Intangibles & 2,000 & 2,000 & 0 & $0 \%$ \\
\hline Activo diferido & & & & \\
\hline Activos no corrientes & 373,339 & 317,797 & 55,542 & $17 \%$ \\
\hline Total activos no corrientes & $1,388,809$ & 847,483 & 541,326 & $64 \%$ \\
\hline
\end{tabular}

\section{TOTAL ACTIVO NETO}

\begin{tabular}{lcccc} 
PASIVO & $\mathbf{2 0 2 0}$ & $\mathbf{2 0 1 9}$ & & \\
\hline Tributos y aportaciones por pagar & 2,440 & 36,812 & $-34,372$ & $-93 \%$ \\
Remuneraciones por pagar & 1,023 & 5,600 & $-4,577$ & $-82 \%$ \\
Anticipos a clientes & $-1,527$ & 298,428 & $-299,955$ & $-101 \%$ \\
Cuentas por pagar comercial & 27,753 & 306,809 & $-279,056$ & $-91 \%$ \\
Obligaciones financieras & $4,873,463$ & 204,135 & $4,669,328$ & $2287 \%$ \\
\hline TOTAL PASIVO & $\mathbf{4 , 9 0 3 , 1 5 2}$ & $\mathbf{8 5 1 , 7 8 4}$ & $4,051,368$ & $476 \%$
\end{tabular}




\begin{tabular}{lcccc} 
PATRIMONIO & $\mathbf{2 0 2 0}$ & $\mathbf{2 0 1 9}$ & & \\
\hline Capital & 500,000 & 500,000 & 0 & $0 \%$ \\
Resultados acumulados & $1,866,706$ & $1,167,090$ & 699,616 & $60 \%$ \\
Utilidad del ejercicio & 844,948 & 968,158 & $-123,210$ & $-13 \%$ \\
\hline TOTAL PATRIMONIO & $\mathbf{3 , 2 1 1 , 6 5 4}$ & $2,635,248$ & 576,406 & $22 \%$ \\
& & & & \\
\hline Total pasivo y patrimonio & 8114806 & 3487032 & $4,627,774$ & $133 \%$ \\
\hline
\end{tabular}

Las cuentas por cobrar comerciales disminuyeron del 2019 al 2020 en un 51\%, se puede apreciar también en el estado de situación financiera el incremento de inmueble, maquinaria y equipo en un $93 \%$ para el periodo siguiente. Con respecto a los subproductos y desechos disminuyo en un $78 \%$ del 2019 al 2020, por otro lado, la mercadería extraída aumento en un 2\%. Las cuentas comerciales disminuyeron en un $91 \%$ del 2019 al 2020,lo cual se refleja en la disminución de mercadería en el periodo 2020. Las obligaciones financieras aumentaron para el periodo 2020 en $2287 \%$ por el préstamo financiero de reactiva Perú. En el caso del patrimonio mantiene la misma cifra para el 2020. El total pasivo mas patrimonio se incrementó en un 133\% para el periodo 2020.

\section{Tabla 14}

Análisis horizontal del estado de resultado

A. Horizontal 20192020

\begin{tabular}{|c|c|c|c|c|}
\hline & & & & $0<0$ \\
\hline & 2020 & 2019 & variación & Porcentaje \\
\hline Ingresos de operación & 25862513 & 21495589 & $4,366,924$ & $20 \%$ \\
\hline (-) Costo de ventas & $24,296,524$ & $20,139,248$ & $4,157,276$ & $21 \%$ \\
\hline
\end{tabular}

\begin{tabular}{ccccc}
\hline Resultado bruto (utilidad) & 1565989 & 1356341 & 209,648 & $15 \%$ \\
\hline Gastos de operación & & & & \\
Gastos de venta & 274,854 & 201,563 & 73,291 & $36 \%$ \\
Gastos de administración & 410,777 & 181,233 & 229,544 & $127 \%$
\end{tabular}

\begin{tabular}{lcccc}
\hline Resultado de operación & 880358 & 973545 & $-93,187$ & $-10 \%$ \\
\hline Gastos financieros & 119,476 & 37,621 & 81,855 & $218 \%$ \\
Ingresos financieros & 48,447 & 12,033 & 36,414 & $303 \%$ \\
Otros ingresos gravados & 35,620 & 20,200 & 15,420 & $76 \%$
\end{tabular}




\begin{tabular}{lcccc}
\hline Resultados antes de impuestos & 844948 & 968158 & $-123,210$ & $-13 \%$ \\
\hline Distribución legal de la renta & 0 & & & \\
Resultados antes de impuestos & 844,948 & 968,158 & $-123,210$ & $-13 \%$ \\
Adiciones para determinar la renta & 34,796 & & 34,796 & \\
Adiciones para determinar la renta & & & & \\
\hline Renta neta imponible & & & & $-88,414$ \\
\hline \hline
\end{tabular}

Los ingresos de operación aumentaron del periodo 2019 a 2020 en un 20\%, se evidencia que los gastos de ventas incrementaron en un 36\% de un periodo a otro, y los gastos de administración en un 127\%. La utilidad operativa disminuyó en un 10\% del 2019 al 2020 y la utilidad neta disminuyo en un $9 \%$.

\section{Discusión}

El condicionante de la indagación fue determinar la relación entre la gestión de capital de trabajo y la toma de decisiones en la empresa Alba Mayo SRL, Moyobamba, 2021. Y con respecto al objetivo general se contempla la relación entre la gestión de capital de trabajo y la toma de decisiones en la empresa Alba Mayo SRL, Moyobamba, 2021. Mediante el estudio estadístico de coeficiente de Pearson se obtuvo un coeficiente de 0. 353 (correlación positiva baja) y un $\mathrm{p}$ valor igual a 0,000 ( $\mathrm{p}$-valor $\leq 0.01$ ) en todas las similitudes, por lo que, se acepta la hipótesis alterna, señala que si existe relación entre la gestión de capital de trabajo y la toma de decisiones en la empresa Alba Mayo SRL, Moyobamba, 2021. Este resultado se asemeja al de Siancas, L. (2020), en su investigación, estudio de los estados financieros y su acontecimiento en la toma de decisiones corporation intherpharma S.A.C. Los resultados de la indagación muestran que los directivos toman decisiones sin hacer su respectivo análisis de ningún método a los estados financieros, para el año 2019 los gastos administrativos aumentaron a $52.75 \%$ y las ventas disminuyeron un $3.65 \%$. Concluyó sobre la observación de los estados financieros influye de manera favorable en la toma de decisiones.

Se coteja el paralelismo de capital de trabajo en la toma de decisiones en la empresa Alba Mayo SRL, Moyobamba, 2021, ya que según el estudio estadístico de Pearson se logró un coeficiente de 0.379 y 0.328 (correlación positiva baja) y un p valor igual a 0,000 (p- 
valor $\leq 0.01)$ en todas las correlaciones, con respecto al nivel de capital de trabajo, el 53\% manifestó que si existe un buen capital de trabajo y el $47 \%$ indicaron que no existe un buen capital de trabajo. Esto se relaciona con la investigación de Arreiza Pusma, E. \& Gavidia Mamani, J. N. (2020), en su revista científica titulada estrategia financiera la gestión de capital de trabajo en el desarrollo empresarial, su metodología de investigación fue aplicada y los resultados mostraron que el capital de trabajo permite asegurar que la empresa cuente con el flujo de caja suficiente para realizar las operaciones normales. Quien nos indica que se debe estimar el tiempo promedio requerido para recuperar las cuentas posibles, lo que equivale a la conversión de productos de cuentas por cobrar en normal, actividades operativas y está directamente relacionada con el flujo de caja de la empresa.

Se contempla la influencia de los activos y pasivos corrientes en la toma de decisiones de la empresa Alba Mayo SRL, Moyobamba, 2021. Mediante el estudio detallado de Pearson se logró un factor de 0.379 y 0.328 (correlación positiva baja) y un p valor igual a 0,000 ( $p$-valor $\leq 0.01)$ en el total de las correlaciones, por ende, se admite la hipótesis alterna, es consecuencia, el nivel de los activos y pasivos corrientes influye favorablemente en la toma de decisiones en la empresa Alba Mayo SRL, Moyobamba, 2021. Silvestre, G. (2021). En su investigación, capital de trabajo y ventaja competitiva de la sociedad consultora y mercancías generales Conserje S.R.L, tesis de pregrado, Universidad Nacional Misiones. Tuvo como resultado que en la empresa el $62.5 \%$ de los colaboradores perciben al capital de trabajo como bueno por ende la empresa es eficiente, con respeto a la ventaja competitiva el $62.5 \%$ encuestados refieren que la empresa si tiene una ventaja competitiva de la empresa. Concluye que entre el capital de trabajo y la ventaja competitiva existe una relación significativa.

Finamente se determinó si el estudio de los estados financieros influye en la toma de disposiciones en la compañía Alba Mayo SRL, Moyobamba, 2021, los resultados muestran que por el análisis detallado de Pearson se alcanzó un coeficiente de 0.323, correlación positiva baja y un p valor igual a 0,000 ( $\mathrm{p}$-valor $\leq 0.01$ ), en todas las correlaciones, por lo que, se acepta la hipótesis alterna, lo que quiere decir es que el estudio de los cambios financieros influyente favorablemente en la toma de disposiciones en la empresa Alba Mayo SRL, Moyobamba, 2021. Esto se relaciona con la investigación de Peña Suarez (2019), en su revista científica titulada el estudio económico como un 
instrumento básico para la toma de decisiones de la compañía Comfalasdi Cia. Ltda, su metodología de investigación fue aplicada, y los resultados mostraron que la sociedad tiene sus pasivos a breve plazo muy elevados lo que implica que la empresa no cuente con liquidez suficiente para afrontar sus obligaciones, concluyó que es muy importantes hacer un análisis financiero ya que este permite conocer cuáles son las deficiencias que tiene la empresa y que al mismo tiempo ayuda a tomar buenas decisiones, esta investigación se relaciona de modo que el autor emplea la misma metodología de investigación que es aplicada y se concluye ambas investigaciones que el análisis de los estados financieros si influyen en la toma de decisiones.

\section{CONCLUSIÓN O CONSIDERACIONES FINALES}

1. Se concluye que si existe relación entre la gestión de capital de trabajo y toma de decisiones en la empresa Alba Mayo S.R.L, contrastada por una significancia de 0,000 y un coeficiente de Pearson en la cual alcanzo un coeficiente de 0.353 la cual es positiva y baja. Muestra una relación directa entre ambas variables, por ende, conocer y definir correctamente los componentes del capital de trabajo sirve para tomar decisiones eficientes y oportunas con el principal objetivo de generar mayor valor a la empresa.

2. En el diagnostico en el nivel de capital de trabajo en la toma de decisiones en la empresa Alba Mayo S.RL, Moyobamba, 2021, según la encuesta el 53\% manifestaron que si existe un buen capital de trabajo y que solo el $47 \%$ manifiestan que no existe un buen capital de trabajo. A través de cálculo del ratio de capital de trabajo se muestra que en el periodo 2019 la empresa dispuso de un capital de trabajo de s/.1,787,765.00 y para el año 2020 obtuvo de s/. 1,822,845.

3. El nivel de los activos corrientes y pasivo corriente influyen favorablemente en la toma de decisiones en la empresa Alba Mayo S.R.L, situación que ha sido contrastada a través de un coeficiente de 0.000 y mediante el análisis estadístico de Pearson de 0.379 y 0.328 la cual fue positiva y baja. También a través de los análisis vertical y horizontal realizado a los 2 periodos se pudo analizar que su pasivo del periodo 2019 tuvo un gasto financiero de S/.204,135 y el periodo 2020 S/.4,873,463 incrementando este último en un 54\%, debido al financiamiento de reactiva Perú. 
4. Se concluye que el análisis de los estados financieros influye favorablemente en la toma de decisiones en la empresa Alba Mayo S.R.L, el cual ha sido contrastada a través de una significancia de 0.000 y una correlación de Pearson de 0.323 que fue positiva y baja. De acuerdo al cálculo ya análisis de ratios realizados a los estados financieros del periodo 2019 y 2020, los gerentes y personal administrativo de la empresa, han podido optar por mejores decisiones que han ido contribuyendo favorablemente en el desarrollo económico de la empresa.

\section{LISTA DE REFERENCIAS}

Aguilar, J. (2020). Estudio de la toma de decisiones en las pequeñas y medianas empresas del sector productor químico de la ciudad de Quito, tesis de pregrado, Universidad Técnica de

Ambato. https://bibdigital.epn.edu.ec/bitstream/15000/20684/1/CD\%2010187.pdf

Alvarado, S. y Zelaya. S. (2018) Información financiera para la toma de decisiones del personal administrativo de una institución financiera peruana, primer bimestre 2018” (tesis de pregrado). Universidad Peruana Unión

Arreiza Pusma, E., \& Gavidia Mamani, J. N. (2020). Working capital management as a financial strategy for business development; Gestión del capital de trabajo como estrategia financiera para el desarrollo empresarial. Revista de Investigación Valor Contable; Vol. 6 Núm. 1 (2019): Revista Científica Valor Contable; 65 - 77.

Betancourt, B. (2017). Análisis sectorial y competitividad. ECOE Ediciones.

Carlos canto. (2019). Importancia de la Gestión del Capital de Trabajo. https://es.linkedin.com/pulse/importancia-de-la-gesti\%C3\%B3n-del-capitaltrabajo-carlos-canto

Chiavenato, I. (2012). Administración en los nuevos tiempos. McGraw-Hill.

Chaves, M., Vallejos, C. (2017). Gestión de la información financiera y su relación con la toma de decisiones gerenciales en las organizaciones de la Unión Peruana del Norte, revista científica, Universidad Peruana Unión.

Duarte, J. y Fernández, L. (2016). Finanzas corporativas: editorial Limusa S.A. de C.V. Grupo Noriega Editores.

Espinosa, C. (2016). ¿Cómo se toman las decisiones organizacionales? Una revisión clásica. Ambiente \& $\quad$ Sociedade, $\quad 31(87), \quad$ 43-78. 
http://www.scielo.org.mx/scielo.php?script=sci_arttext\&pid=S0187-

01732016000100002

Guerras, L., Navas, J. (2016). La dirección estratégica de la empresa teoría y aplicaciones. Ediciones Aranzadi, S.A.

Guzmán, M. d. (2018). Toma de decisiones en la gestión financiera para el sistema empresarial (Primera ed.). Editorial Grupo Compás. http://142.93.18.15:8080/jspui/bitstream/123456789/278/1/LIBRO\%20LISTOilovepdf-compressed-2.pd

Harvard Business Review (2017). Guías HBR, Finanzas Básicas. Reverté Management. Herz, J. (2016). Apuntes de contabilidad Financiera. Universidad de Ciencias Aplicadas (UPC).

Herrera, A., Vega, S., Vivanco, E., \& Herrera, A. (2016). Razones financieras de liquidez en la gestión empresarial para la toma de decisiones. Revista de la Facultad de Ciencias Contables, 24(46), 151-160. doi: https://doi.org/10.15381/quipu.v24i46.13249

Hernández y Mendoza (2018). Metodología de la investigación científica. Marcos.

Hernández et al. (2018) Pasos para elaborar proyectos de investigación científica: Cuantitativa, cualitativa y mixta. Editorial San Marcos E.I.R.L.

Lira Briceño, P. (2016). Apuntes de finanzas cooperativas. Universidad Peruana de Ciencias Aplicadas (UPC).

Mallo, C. y Rocafort, A. (2016) “Contabilidad de Costes”. 33 Edición. Editorial Pirámide. Malquin, D. M. S., \& Chamorro, y M. G. E. (2019). Presupuesto De Capital De Trabajo Para La Empresa “Transcomerinter” De La Ciudad De Tulcán. Investigación Operacional, 40(4), 496

Muralikrishna, B. (2012). Working capital management. Lovely Professional University Phagwara.

Ortiz, H. (2015). Análisis financiero aplicado y normas internacionales de información financiera - NIIF. Universidad Externado de Colombia.

Otzen y Manterola. (2017). Técnicas de Muestreo sobre una Población a Estudio. Int. J. Morphol., 35(1):227-232, 2017. 
Paredes, E. (2016). La toma de decisiones y su incidencia en la ventaja competitiva en la empresa Mondelēz International, Chiclayo - 2016. tesis de pregrado. Universidad César Vallejo.

Paola, C. (2019). Capital de trabajo y su incidencia en la ventaja competitiva de las Mypes ferreteras, Comas, 2018. Tesis de pregrado. Universidad Cesar Vallejo

Pérez, C., Rodríguez, M, \& Armas, G. (2013). Estrategia como herramienta de gestión. México: Alfaomega grupo editor S.A.

Peña Suárez, D., Escobar Campoverde, N. S., Navas Espín, G. R., \& Portero López, P. R. (2019). Análisis financiero como herramienta básica en la toma de decisiones de la empresa Comfalasdi Cía. Ltda. “Graiman”. (Spanish). Dilemas Contemporáneos: Educación, Política y Valores, 6, 1-16.

Rivera, X. (2014). Calidad del servicio al cliente y su incidencia en la ventaja competitiva de las empresas del sector de la pintura en la ciudad de Guayaquil. (tesis de pregrado) Universidad Católica de Santiago de Guayaquil.

Rivillas, C. I. S., Escobar, J. M., \& Londoño, A. L. R. (2017). Diagnóstico financiero de las empresas en etapa temprana en antioquia. Criterio Libre, 15(26), 131-155. https://www.proquest.com/scholarly-journals/diagnóstico-financiero-de-lasempresas-en-etapa/docview/2125260512/se-2?accountid=37408

Rosell, C. (2019). Relación entre el análisis e interpretación de estados financieros y la toma de decisiones financieras en las pequeñas empresas mineras de Chile, revista científica, Universidad de Chile.

Rojas, R. (2014). Investigación Social. México: Plaza y Valdés. https://raulrojassoriano.com/cuallitlanezi/wp-content/themes/raulrojassoriano

Sanabria, G., Muñes, F. (2017). La probabilidad como elemento orientador de la toma de decisiones. Tesis de pregrado. Universidad Técnica de Ambato - Ecuador.

Sánchez, A. (2018). 7 pasos para una tesis exitosa, Desde la idea inicial hasta la sustentación. Universidad de San Martín de Porres.

Sebastián Jaramillo Aguirre. (2016). Relación entre la gestión del capital de trabajo y la rentabilidad en la industria de distribución de químicos en Colombia. Revista Finanzas y Política Económica.

Siancas, L. (2020). Análisis de los estados financieros y su incidencia en la toma de decisiones, Corporation Intherpharma s.a.c.-Lima, años 2017, 2018 y 2019. tesis 
para obtener el título profesional de contador público, Universidad Privada del Norte.

https://repositorio.upn.edu.pe/bitstream/handle/11537/25546/Siancas\%20Centuri on $\% 2 \mathrm{c} \% 20$ Lucila\%20Elizabeth.pdf?sequence=1\&isAllowed $=\mathrm{y}$

Tanaka, G. (2015). Contabilidad y análisis financiero: un enfoque para el Perú. Fondo Editorial de la Pontificia Universidad Católica del Perú.

Tipán, E. (2015). El capital de trabajo y su incidencia en la rentabilidad de la empresa Soluciones integrales en redes seguridades y comunicaciones Sinfotecnia en el año 2013 (tesis de pregrado). Universidad Técnica de Ambato - Ecuador.

Torrejón, K. (2016). Financiamiento de capital de trabajo y su incidencia en el desarrollo de las Mypes textiles en el distrito de la Victoria, año 2015 (tesis de pregrado). Universidad Cesar Vallejo.

Van Horne, J. y Wachowicz, J. (2016). Fundamentos de Administración Financiera. Pearson Educación de México.

Vanauken, Howard E.; Ascigil, Semra; and Carraher, Shawn (2017) "Turkish SMEs' Use of Financial Statements for Decision Making," The Journal of Entrepreneurial Finance: Vol. $\quad$ 19: Iss. https://digitalcommons.pepperdine.edu/jef/vol19/iss1/6 